Please do not remove this page

RMIT

UNIVERSITY

\title{
Acoustically tunable optical transmission through a subwavelength hole with a bubble
}

Maksymov, Ivan; Greentree, Andrew

https://researchrepository.rmit.edu.au/esploro/outputs/9921861806201341/filesAndLinks?institution=61RMIT_INST\&index=null

Maksymov, I., \& Greentree, A. (2017). Acoustically tunable optical transmission through a subwavelength hole with a bubble. Physical Review A, 95(3), 1-10. https://doi.org/10.1103/PhysRevA.95.033811

Document Version: Published Version

Published Version: https://doi.org/10.1103/PhysRevA.95.033811

Repository homepage: https://researchrepository.rmit.edu.au

(C) 2017 American Physical Society

Downloaded On 2023/04/26 22:01:37 +1000

Please do not remove this page 
Thank you for downloading this document from the RMIT Research Repository.

The RMIT Research Repository is an open access database showcasing the research outputs of RMIT University researchers.

RMIT Research Repository: http://researchbank.rmit.edu.au/

\section{Citation: \\ Maksymov, I and Greentree, A 2017, 'Acoustically tunable optical transmission through a subwavelength hole with a bubble', Physical Review A, vol. 95, no. 3, 033811, pp. 1-10.}

See this record in the RMIT Research Repository at:

https://researchbank.rmit.edu.au/view/rmit:43734

Version: Published Version

Copyright Statement:

(C) 2017 American Physical Society

Link to Published Version:

https://dx.doi.org/10.1103/PhysRevA.95.033811 


\title{
Acoustically tunable optical transmission through a subwavelength hole with a bubble
}

\author{
Ivan S. Maksymov and Andrew D. Greentree \\ ARC Centre of Excellence for Nanoscale BioPhotonics, School of Science, RMIT University, Melbourne, VIC 3001, Australia
}

(Received 24 October 2016; published 13 March 2017)

\begin{abstract}
Efficient manipulation of light with sound in subwavelength-sized volumes is important for applications in photonics, phononics, and biophysics, but remains elusive. We theoretically demonstrate the control of light with MHz-range ultrasound in a subwavelength, 300-nm-wide water-filled hole with a 100-nm-radius air bubble. Ultrasound-driven pulsations of the bubble modulate the effective refractive index of the hole aperture, which gives rise to spectral tuning of light transmission through the hole. This control mechanism opens up novel opportunities for tunable acousto-optic and optomechanical metamaterials, and all-optical ultrasound transduction.
\end{abstract}

DOI: 10.1103/PhysRevA.95.033811

\section{INTRODUCTION}

The presence of a single circular hole with the diameter $w$ in an opaque metal film, with $w$ much smaller than the wavelength of incident light $\lambda_{0}$, leads to optical phenomena unpredicted by the classical aperture theories [1-3]. Such phenomena include enhanced transmission of light through the hole [1-3], Purcell effect and emission of nonclassical light [4,5], as well as spectroscopy and sensing [6]. These functionalities are achievable in both single and periodically arrayed holes, and they are due to the interaction of light with surface plasmon resonances at the surface of the metal film and Fabry-Perot resonances of guided optical modes inside the hole [1-3].

The Fabry-Perot resonances give rise to a peak in light transmission through a single hole when $\lambda_{0} \approx \lambda_{\mathrm{c}}$, where $\lambda_{\mathrm{c}} \propto w n_{\mathrm{f}}$ is the cutoff wavelength of the fundamental guided mode of the hole and $n_{\mathrm{f}}$ is the refractive index of the material filling the hole $[3,4]$. Thus, the transmission peak becomes spectrally tunable by either changing $w$ or controlling $n_{\mathrm{f}}$ $[2,3]$. This has been achieved by using stretchable metal nanovoids [7], liquid crystals [8], nonlinear optical materials [9], and electrically tunable semiconductor materials [10]. Spectral tuning of transmission has also been demonstrated by applying external magnetic fields [11] and using surface acoustic waves [12].

Other types of electromagnetic waves, e.g., microwaves, may also be transmitted through subwavelength apertures in a fashion similar to light [13]. Enhanced transmission of sound through acoustically subwavelength apertures has also been observed [14-16].

The coexistence of the waves of different nature in the same structure allows us to control one wave with another (e.g., light in holes filled with a magnetodielectric material may be controlled with microwaves and vice versa [17]). However, despite the progress in the control of the interaction of light with structural deformations of micro- and nanostructures $[18,19]$, spectral tuning of the optical transmission through subwavelength apertures with ultrasound remains elusive.

In this work we theoretically demonstrate a spectral tuning of light transmission with sound in a subwavelength-sized volume. A water-filled, 300-nm-wide circular hole in a 400-nm-thick silver film acts as a subwavelength and deep subwavelength aperture for light and ultrasound, respectively. A spherical air bubble [20-22] with the $100 \mathrm{~nm}$ at-rest radius is trapped and stabilized inside the hole [Fig. 1(a)], which may be achieved by using a variety of techniques [21-31]. In general, the bubble maintains its sphericity when it harmonically pulsates, at the microsecond scale, in response to ultrasound that evanescently enters and leaks into the hole. By solving the Rayleigh-Plesset equation of the bubble dynamics [20,21], we demonstrate that the pulsations of the bubble allow for the tuning of light transmission as a function of the ultrasound pressure. This tuning mechanism opens up novel opportunities for photonics and phononics, such as all-optical ultrasound transduction at the subwavelength scale and tunable acousto-optic and optomechanical metamaterials.

\section{ACOUSTICALLY TUNABLE OPTICAL TRANSMISSION}

There are a number of techniques that may be used to generate and trap a bubble inside subwavelength apertures such as nanopores and nanochannels [21-31]. Although the exact mechanisms of the bubble formation and trapping inside nanoporous materials are yet to be confirmed, there exist commercial devices that can trap and count nanometer-size bubbles and particles [30,31]. In certain embodiments of such devices, only a single nanopore is used, which makes them of immediate relevance to our discussion. It has also been shown that nanopores may be combined with a metal nanocavity that supports plasmon modes [28]. In such plasmonic nanopores, a plausible mechanism of the generation and trapping of bubbles is the plasmon-induced Joule heating. Thus, the bubble may potentially be generated and trapped inside the hole by all-optical means.

As the bubble pulsates inside the hole, the optical cutoff wavelength $\lambda_{\mathrm{c}}$ of the hole changes from $\sim 1.7 w n_{\text {water }}$ for the entire hole filled with water to reach the asymptotic value $\sim 1.7 w n_{\text {air }}$ for the air-filled hole [2-4], being $n_{\text {water }}=1.33$ and $n_{\text {air }}=1$. Thus, for $w=300 \mathrm{~nm}$ the transmission will be tuned from $\sim 700 \mathrm{~nm}$ (at the lowest bubble radius) to $\sim 510 \mathrm{~nm}$ (at the largest radius), which is confirmed by three-dimensional (3D) finite-difference time-domain (FDTD) simulations (solid and dashed curves in Fig. 2). Numerical details of the FDTD method are discussed in the Appendix.

For a spherical bubble in an unbounded liquid, the pulsation implies a variation in the bubble volume, defined by the radius of the bubble $R(t)$ that harmonically varies around its at-rest radius $R_{0}$. In real systems, the pulsations are damped due to 


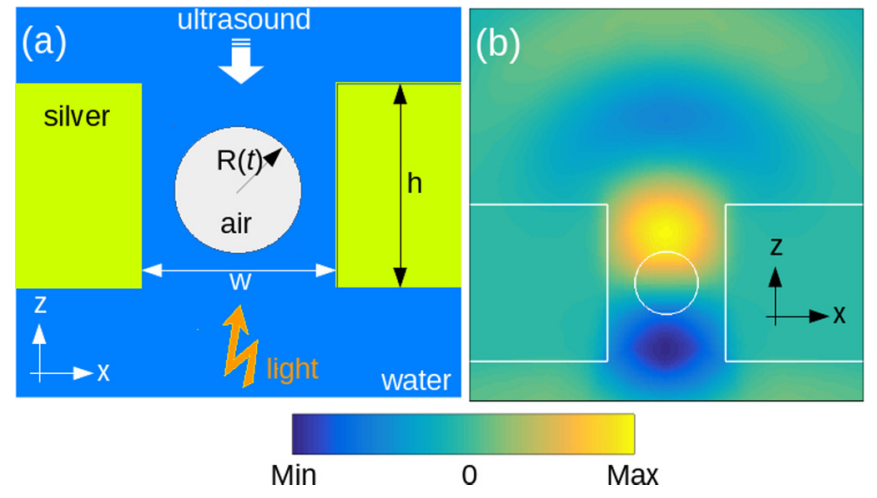

FIG. 1. (a) Schematic cross section of the water-filled hole with an air bubble. The thickness of the silver thin film is $h=400 \mathrm{~nm}$. The diameter of the hole is $w=300 \mathrm{~nm}$. The wavelength of ultrasound is two orders of magnitude larger than $w$. (b) Instantaneous $E_{\mathrm{x}}$ electric field amplitude snapshot in the cross section of the hole with a bubble. The incident optical plane wave propagates in the $+z$ direction.

acoustic losses, action of viscous stresses at the gas-liquid interface, thermal conduction across the interface [21,32], and other phenomena. The effect of these dissipation mechanisms is discussed in Sec. III. Until that point, we will neglect losses in our analysis because our main goal is to demonstrate the effect of acoustically tunable optical transmission through the hole.

Thus, we solve the following Rayleigh-Plesset equation that models a bubble in an inviscid and incompressible liquid:

$$
\rho\left(\ddot{R} R+1.5 \dot{R}^{2}\right)=p_{0}\left(R_{0} / R\right)^{3 \gamma}-p_{0}-P(t) .
$$

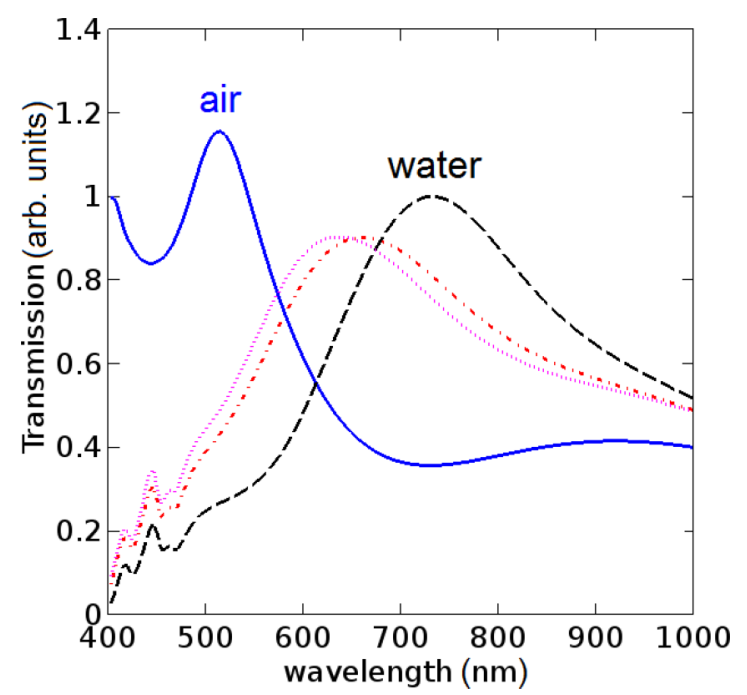

FIG. 2. Optical transmission through the hole. Blue solid curve: The hole is filled with air. Black dashed curve: The hole is filled with water. Magenta dotted curve: The hole is filled with water and an $R_{0}=100 \mathrm{~nm}$ air bubble. Red dashed-dotted line: The hole is filled with water and an ellipsoidal air bubble with $R_{0, \mathrm{x}}=R_{0, \mathrm{y}}=$ $100 \mathrm{~nm}$ and $R_{0, \mathrm{z}}=80 \mathrm{~nm}$ at-rest radii (see Fig. 1 for the coordinate framework). All curves are normalized to the maximum transmission value for the water-filled hole.

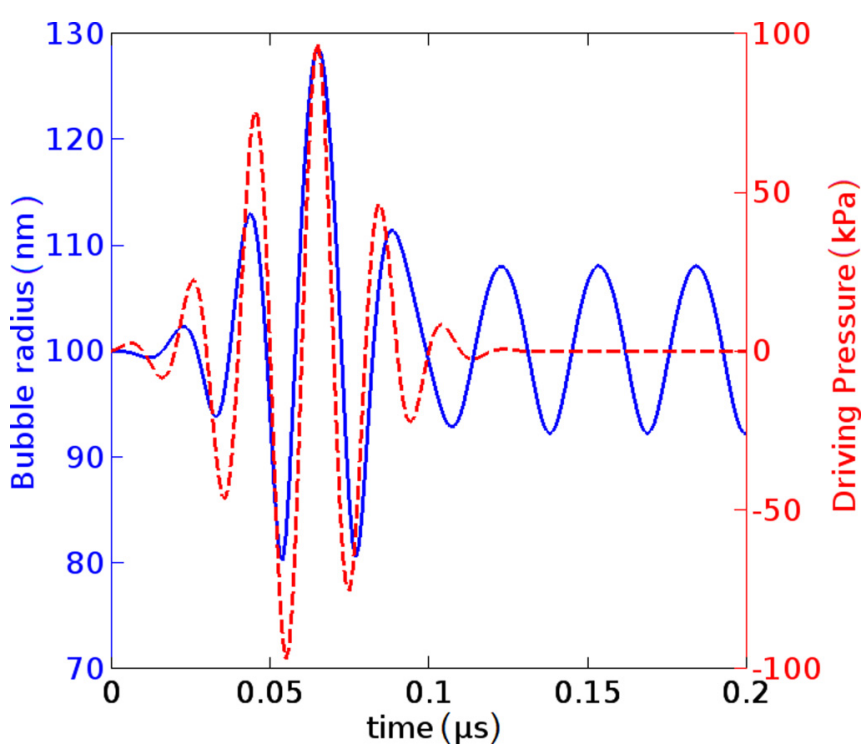

FIG. 3. Radial response $R(t)$ of a 100-nm-radius air bubble in water (blue solid curve) to the driving ultrasound pressure pulse $P(t)$ with the center frequency $50 \mathrm{MHz}$ (red dashed curve). Note that this result is obtained in the lossless approximation, which explains the resonant pulsations of the bubble at $t>0.1 \mu \mathrm{s}$.

The initial values are $R_{0}=100 \mathrm{~nm}$ and $\dot{R}=1 \mathrm{~m} / \mathrm{s}[20,21]$. The constant reference pressure is $p_{0}=100 \mathrm{kPa}$, the density of water is $\rho=1000 \mathrm{~kg} / \mathrm{m}^{3}$, and the polytropic exponent of air is $\gamma=1.4[21,33]$. It is noteworthy that Eq. (1) is known to produce a good agreement with experiments on both micronsize and submicron bubbles (nanobubbles) [21,34-36].

In our analysis, in Eq. (1) the driving pressure $P(t)$ (red dashed curve in Fig. 3) is a Gaussian-enveloped sinusoid with the frequency $f=50 \mathrm{MHz}$, which is detuned from the linear resonance frequency $f_{0} \approx 30 \mathrm{MHz}$ of the bubble in water $\left(f_{0} R_{0} \approx 3 \mathrm{~m} / \mathrm{s}[20,21]\right)$. This detuning allows us to avoid elevated acoustic forcing of the bubble to always keep $R(t)<$ $w / 2$. Since the bubble is securely trapped inside the hole, we neglect the feedback between the pulsations and translational motion of the bubble $[33,37]$.

Figure 3 (blue solid curve) shows that $R(t)$ varies in response to the driving pressure $P(t)$ with a phase lag due to the inertia of the surrounding water $[20,21]$. The maximum (minimum) value of $R(t)$ reached by the bubble is $\sim 130 \mathrm{~nm}$ $(\sim 80 \mathrm{~nm})$. When $P(t)=0$, the bubble continues pulsating with a smaller amplitude because damping is not taken into account in Eq. (1). However, in Sec. III we show that this resonant tail disappears when acoustic losses are taken into account.

By using the Og̃uz and Prosperetti theory [32] we show that the pulsation frequency of the bubble inside the rigid water-filled hole is $\sim 2$ times smaller than that in unbounded water $f$. This theory assumes that inside a water-filled rigid circular tube a pulsating bubble maintains a spherical shape but its pulsation frequency $f_{\text {tube }}$ is defined as

$$
\left(\frac{f_{\text {tube }}}{f}\right)^{2}=\frac{R_{\text {tube }}^{2}}{4 R_{0}}\left(\frac{1}{L_{1}^{e}}+\frac{1}{L_{2}^{e}}\right),
$$



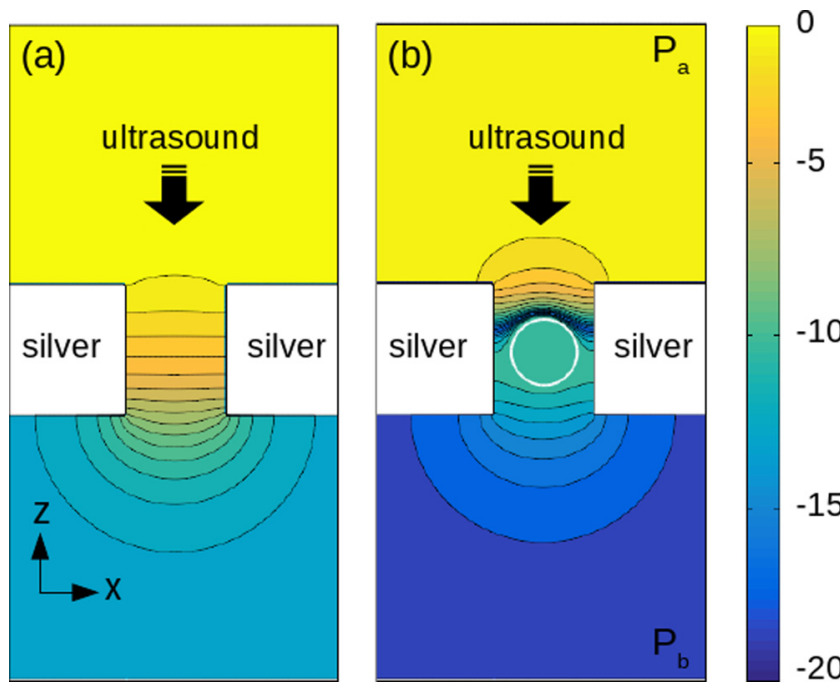

FIG. 4. (a) Ultrasound pressure profile (dB-scale color bar) in the vertical cross section of the hole without the bubble. (b) The same as in (a) but the hole is loaded with a 100-nm-radius bubble (open circle). Both results are obtained in the lossless approximation.

where $L_{\mathrm{i}}^{e}=L_{\mathrm{i}}-h / 2+\Delta L(\mathrm{i}=1,2)$ is the bubble position inside the tube and the at-rest radius of the bubble $R_{0}$ is comparable with, but smaller than the radius of the tube $R_{\text {tube }}$.

In the derivation of Eq. (2), the spherical bubble was replaced with a cylindrical one with the same volume, occupying the entire cross section of the tube. The thickness $h$ of the cylindrical bubble enters the expression for $L_{\mathrm{i}}^{e}$. This replacement simplifies the system to one-dimensional motion, where the bubble provides the effective stiffness and the two liquid columns to either side of the tube provide the effective inertia, which allows us to determine the natural frequency of the resulting spring-mass system [32,37]. The correction factor $\Delta L=0.62 R_{\text {tube }}$ accounts for the inertia of the liquid outside the tube [32].

In experiments in [37], the ratio of bubble radius to tube radius is $R_{0} / R_{\text {tube }}=0.55-0.8$. Following the discussions above, we assume that it is adequate to use Eq. (2) and the results from [37] to describe the behavior of nanoscale bubbles. By substituting $R_{\text {tube }}$ with the radius of the hole, we obtain $R_{0} / R_{\text {hole }}=0.66$, which lies within the experimentally verified range. Finally, we obtain the pulsation frequency of the bubble inside the hole $f_{\text {hole }} \approx 0.48 f$.

The wavelength of $f=50 \mathrm{MHz}$ ultrasound is $\lambda_{\mathrm{a}}=30 \mu \mathrm{m}$ (the speed of sound in water is $v_{\text {water }}=1500 \mathrm{~m} / \mathrm{s}$ ), which is 100 times larger than $w$. Thus, the hole operates in an acoustically deep subwavelength regime, which was not the focus of the previous relevant works [14-16]. It is instructive to demonstrate that ultrasound may still be coupled to the hole despite the fact that the cutoff frequency $f_{\mathrm{c}}=1.842 v_{\text {water }} /(\pi w)$ [38] of the fundamental guided mode of the hole is $\sim 60$ times larger than $f$.

We simulate the two scenarios of ultrasound normally incident from above the water-filled hole without [Fig. 4(a)] and with the air bubble [Fig. 4(b)]. We use a 3D acoustic FDTD method that models the pressure waves in water and air. However, because of a large mismatch between the characteristic specific acoustic impedance of water and that of silver, the silver film is modeled as a perfectly rigid object [20].

The perfectly rigid body approximation significantly simplifies the simulation, but at the same time it allows us to obtain a qualitatively correct physical picture of ultrasound leakage into a single deep-subwavelength hole. Indeed, a previous analytical theory [39] demonstrates that ultrasound is transmitted through a single hole in a perfectly rigid film approximately in proportion to the hole area. Thus, we do not expect a high ultrasound transmission through the single deep-subwavelength hole considered in this work, because its area is very small. Furthermore, this theory also demonstrates that the perfectly rigid model of the silver film may mostly be applied to the case of single holes, because hole arrays in perfectly rigid films do not exhibit full ultrasound transmission due to the absence of lattice resonances. Consequently, we stress that the results obtained with the 3D acoustic model are mostly valid for the single holes.

The validity of the perfectly rigid body approximation is additionally confirmed by a 3D elastodynamic FDTD model that takes into account the real material parameters of silver. However, this model requires significant computational efforts as compared with the acoustic FDTD model. We demonstrate (see Appendix) that the elastodynamic model confirms the validity of the result obtained with the acoustic model. We also note that in our acoustic simulations we neglect losses in ultrasound transmission through the hole. The impact of acoustic losses is discussed in Sec. III.

Because $w<<\lambda_{\mathrm{a}}$, ultrasound is reflected from the silver film, which leads to the pressure doubling [38] in the region above the film. For clarity, in Fig. 4(a) the pressure magnitude above the hole is normalized such that $P_{\mathrm{a}}=1$. However, ultrasound also evanescently enters the hole and decays inside it, which leads to a partial transmission of pressure $P_{\mathrm{b}}$ to the region below the hole. Without the bubble we obtain $P_{\mathrm{a}} / P_{\mathrm{b}} \approx 15$. In the middle of the hole, where the bubble would be located, we observe $P_{\mathrm{a}} / P_{\text {bubble }} \approx 2$, which implies that a two times larger driving pressure will be required to compensate for the ultrasound attenuation in the hole. When the bubble is inside the hole [Fig. 4(b)], its surface acts as a highly reflecting pressure release boundary [38], in front of which $P \approx 0$. Nevertheless, we observe that the hole loaded with the bubble remains partially transparent, with $P_{\mathrm{a}} / P_{\mathrm{b}} \approx 50$

Now we discuss the optical transmission and reflection spectra of the hole as a function of the bubble radius (Fig. 5). Because the variations of $R(t)$ are much slower than the transient optical processes in the hole, the bubble is considered to be at rest but its radius takes one of the possible values of $R(t)$ in Fig. 3.

In transmission [Fig. 5(a)], the resonance wavelengths $\lambda_{\text {res }}$ blueshift from $\sim 700$ to $\sim 525 \mathrm{~nm}$ as the radius of the bubble is increased from 80 to $130 \mathrm{~nm}$. The pulsations of the bubble are responsible for the blueshift, because they lead to a change in the cutoff wavelength $\lambda_{\mathrm{c}} \approx \lambda_{\text {res }}$. A representative profile of the fundamental guided mode of the hole is shown in Fig. 1(b). The blueshift of the minima in the reflection spectra [Fig. 5(b)] is effectively smaller than in transmission, because light interacts with the hole either resonantly or nonresonantly, but interference between these two mechanisms gives rise to 

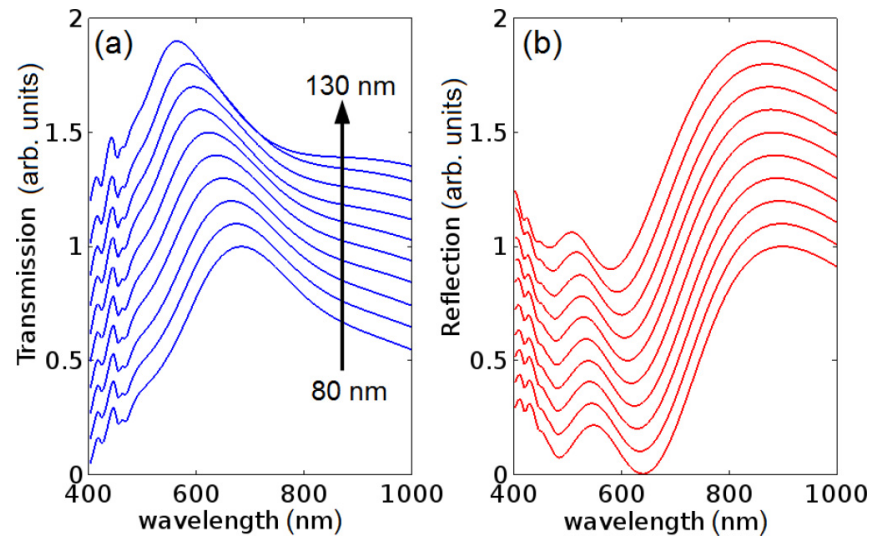

FIG. 5. Transmission (a) and reflection (b) spectra of the hole in the presence of the bubble at rest, plotted as a function of the bubble radius. All curves are normalized to unity and offset along the $y$ axis for the sake of visualization. (a) and (b) The radius increases from 80 to $130 \mathrm{~nm}$ with a $5 \mathrm{~nm}$ increment, as indicated by the vertical arrow in (a).

two differently asymmetric resonance profiles in transmission and reflection [40]. These asymmetries lead to a spectral offset between the corresponding transmission maxima and reflection minima.

Figure 6(a) shows the intensity of light transmitted through the hole at $561 \mathrm{~nm}$, plotted as a function of the bubble radius
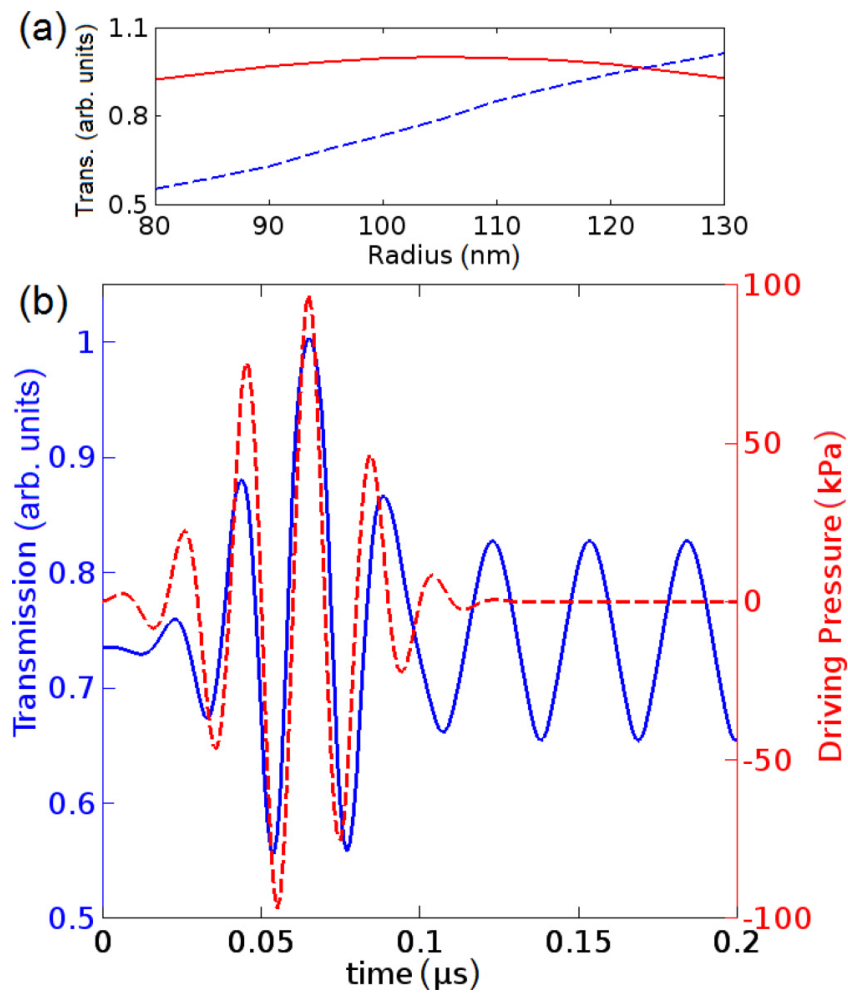

FIG. 6. (a) Transmitted light intensity through the hole at $561 \mathrm{~nm}$ (blue dashed curved) and $632 \mathrm{~nm}$ (red solid curve), plotted as a function of the bubble radius. (b) Transmitted light intensity at $561 \mathrm{~nm}$ as a function of time (blue solid curve). The driving ultrasound pressure $P(t)$ (red dashed curve) can be measured by detecting the intensity. (blue dashed curve). The chosen wavelength corresponds to the transmission peak for the hole with the 130-nm-radius bubble. We observe a quasilinear dependence of the intensity on the bubble radius. At $632 \mathrm{~nm}$ (red solid curve), which corresponds to the peak of the light intensity transmitted through the hole with the 100-nm-radius bubble, we obtain a bell-shaped curve in which the same intensity corresponds to two different radii of the bubble. Thus, at $561 \mathrm{~nm}$ each discrete value of the light intensity can be unambiguously correlated with a unique value of $R(t)$. Also, the transmitted intensity contrast is $\sim 50 \%$ at $561 \mathrm{~nm}$ as compared with $\sim 15 \%$ at $632 \mathrm{~nm}$.

Figure 6(b) (blue solid curve) shows that the monitoring of the light intensity transmitted through the hole at $561 \mathrm{~nm}$ allows us to recover the original line shape of $P(t)$ (red dashed curve). By fitting the resulting curve with the Rayleigh-Plesset equation [Eq. (1)] we can obtain the frequency and amplitude of $P(t)$. This functionality may be used in the optical sensing of ultrasound [41]. Such a liquid-state nanophotonic device may be integrated with the existing liquid-state devices such as, e.g., liquid-core optical fibers [42] and lenses [43]. However, liquidstate photonics still has a number of technical limitations and we anticipate that, for example, to sense ultrasound with a bubble one will need to reduce the impact of the limited detection bandwidth.

Our discussion is expected to be valid when the pulsating bubble becomes aspherical, which can happen, for example, because of the impact of the hole. In general, rigorous numerical simulations with a coupled boundary element method and finite element method (BEM-FEM) have to be conducted to model time-dependent aspherical oscillations of the bubble [37], because there are no analytical or semianalytical theories for a satisfactory prediction of aspherical oscillations.

However, such complex simulations are not required for a qualitative analysis of the dynamic light-bubble interaction and may be avoided by doing analysis in the static regime. We use simulated data for an aspherical air bubble pulsating inside a rigid tube with $\lambda_{\mathrm{a}} / R_{\text {tube }}=150$ [37], where $\lambda_{\mathrm{a}}$ is the wavelength of ultrasound. Because for our hole $\lambda_{\mathrm{a}} / R_{\text {hole }}=$ 200, we expect a similar asphericity in our case. In [37], the pulsating bubble was shown to be more elongated in the axial direction of the tube on expansion and in the radial direction on collapse. The maximum asphericity in the axial (radial) direction was $\sim 25 \%(1 \%-2 \%)$.

Transmission of light through the hole is sensitive to changes in the refractive index and effective volume of the filling material [3]. As compared with the water-filled hole without the bubble, the maximum spectral tuning of the transmission is achieved when the hole is completely filled by an air cylinder [44] (solid and dashed curves in Fig. 2). Furthermore, any intermediate air-filling shape between the sphere and the cylinder, such as an ellipsoid, gives rise to a spectral shift that is close to that produced by a spherical hole with comparable dimensions.

A representative ellipsoidal bubble with the above discussed aspherity from [37] is investigated in Fig. 2 (dasheddotted and dotted curves). We observe a small spectral shift as compared with the case of the perfectly spherical bubble. Thus it appears that bubbles of the other irregular shapes will also produce a similar spectral shift. In particular, we point out that spectral tuning will be achieved with surface nanobubbles and 
nanodroplets [45], which may be created at the solid-liquid interface of the hole.

\section{EFFECT OF ABSORPTION LOSSES}

In the previous discussions, in our acoustic simulations we have neglected losses in ultrasound transmission through the hole, which is a valid approximation for ultrasound propagating in a bulk liquid [46]. However, the presence of viscous and thermal boundary layers [46] in close proximity to the hole's walls no longer warrants this approximation. This is because the thickness of these layers becomes comparable with the diameter of the hole and thus absorption losses cannot be neglected any more [47].

In close proximity to the hole's walls the tangential particle velocity approaches zero, which gives rise to a viscous boundary layer with the thickness $d_{\mathrm{v}}=\sqrt{\eta /(\pi f \rho)}$, where $f$ is the frequency of ultrasound, $\rho$ is the density, and $\eta$ is the viscosity of the liquid. Moreover, heat exchange between the liquid and the walls gives rise to a thermal boundary layer with the thickness $d_{\mathrm{h}}=\sqrt{\kappa /\left(\pi f \rho c_{\mathrm{p}}\right)}$, where $\kappa$ is the thermal conductivity of the liquid and $c_{\mathrm{p}}$ is the corresponding specific heat capacity at constant pressure. Hence, we calculate that at $f=50 \mathrm{MHz} d_{\mathrm{v}} \approx 80 \mathrm{~nm}$ and $d_{\mathrm{h}} \approx 30 \mathrm{~nm}$. Moreover, because the pulsation frequency of the bubble inside the hole is two times smaller than the frequency of the unbounded bubble but the thickness of both viscous and thermal layers scales as $1 / \sqrt{f}$, at $f=25 \mathrm{MHz}$ we obtain $d_{\mathrm{v}} \approx 113 \mathrm{~nm}$ and $d_{\mathrm{h}} \approx 43 \mathrm{~nm}$. That is, in the latter case the thickness of the viscous layer constitutes $\sim 75 \%$ of the hole radius.

To simulate the impact of the viscous and thermal boundary layers, we use the FDTD formalism and the relevant material constants from the Supplementary Information of [41]. Similar to the case of bulk water, we assume that outside the hole the losses are negligibly small, and we simulate the following scenarios: (a) the entire hole volume absorbs ultrasound through a combined viscous and thermal dissipation, (b) absorption only happens near the walls of the hole in a layer with the thickness $d_{\mathrm{v}}=80 \mathrm{~nm}(f=50 \mathrm{MHz})$, and (c) absorption only happens near the walls of the hole in a layer with the thickness $d_{\mathrm{v}}=110 \mathrm{~nm}(f=25 \mathrm{MHz})$.

We note that case (a) represents the worst case scenario in which the total volume of the hole absorbs ultrasound due to the boundary layer effect. Moreover, due to peculiarities of our software implementation we assume that $d_{\mathrm{h}}=d_{\mathrm{v}}$ in cases (b) and (c). This simplification does not interfere with our analysis because in water the contribution of the viscous effects to the total absorption is $\operatorname{Pr}=\mathrm{c}_{\mathrm{p}} \eta / \kappa \approx 7$ times larger as compared with the contribution of the thermal effect, where $\mathrm{Pr}$ is the Prandtl number.

Before we discuss our results, we note that the discussion of the impact of the viscous and thermal boundary layers in [47] is mostly focused on acoustic transmission through subwavelength cavities supporting Fabry-Perot-like resonances. In contrast, in this present work we investigate a deep subwavelength aperture that does not support such resonances but, from the acoustic point of view, supplies ultrasound to the bubble via leakage into the hole.

Previous relevant studies of transmission through a nonresonant system of periodic, very thin screens with air slits demonstrated that viscous losses dramatically reduce the transmission coefficient as compared with the nonviscous case [48,49]. However, those papers, as well as [47], do not investigate the pressure profile inside the aperture. In contrast, in our case we do not require a high transmission coefficient through the hole but we are mostly interested in the pressure profile inside the hole, especially in the region where the bubble would be located.

The results of simulations are shown in Fig. 7. When the entire hole volume is modeled as an absorptive medium [Fig. 7(a)], we observe that the transmission through the hole is low with the characteristic ratio $P_{\mathrm{a}} / P_{\mathrm{b}} \approx 670$. However, in the region where the bubble would be located, i.e., in the middle of the hole, we observe $P_{\mathrm{a}} / P_{\text {bubble }} \approx 55$. Recall that in the lossless approximation we obtained $P_{\mathrm{a}} / P_{\mathrm{b}} \approx 15$ and $P_{\mathrm{a}} / P_{\text {bubble }} \approx 2$. Thus, this result shows that absorption losses have a significant adverse effect on ultrasound transmission through the hole, but overall they do not qualitatively affect the results obtained in the lossless approximationthe ultrasound leaks into the hole and reaches the bubble.

Simulations taking into account the two different thicknesses of the viscous boundary layer [Figs. 7(b) and 7(c)] produce a better result than in the case of the entirely absorbing hole volume: the transmission through the hole is $P_{\mathrm{a}} / P_{\mathrm{b}} \approx 460$ $(\approx 580)$ and $P_{\mathrm{a}} / P_{\text {bubble }} \approx 40(\approx 50)$ at $50 \mathrm{MHz}(25 \mathrm{MHz})$. This implies that due to the impact of the boundary layers one has to increase the peak pressure of the incident ultrasound up to $\sim 2 \mathrm{MPa}$, which is a feasible value [41].

Our simulations also demonstrate that the pressure profiles inside the hole are qualitatively the same at 25 and $50 \mathrm{MHz}$ incident ultrasound frequency. This observation is in good agreement with the conclusions about a broadband nature of the viscous and thermal boundary layers effect drawn in [47]. At the same time, our results are in agreement with the theory from [39] according to which ultrasound is transmitted through the hole approximately in proportion to the hole area. The presence of the viscous and thermal boundary layers effectively decreases the area of the hole and thus for the thicker layers $(25 \mathrm{MHz})$ the transmission through the hole is smaller than for the thinner ones $(50 \mathrm{MHz})$.

Now we discuss the impact of the dissipation mechanisms on the pulsations of the bubble inside the hole. Strictly speaking, in this case we cannot rely on the Og̃uz and Prosperetti theory of the frequency of bubble oscillations inside rigid apertures [32], because this theory was developed for bubbles with $>10 \mu \mathrm{m}$ radii. Indeed, the at-rest radius $R_{0}$ of the bubble considered in this work is two orders of magnitude smaller than $10 \mu \mathrm{m}$.

Nevertheless, the analysis of the limiting cases of the Og̃uz and Prosperetti theory allows us to neglect the acoustic radiation and the losses of thermal origin, because these dissipation mechanisms are dominant only for millimeter-size bubbles [32]. Moreover, it is known that viscous losses are always dominated by the thermal losses except for bubbles with $R_{0}<10 \mu \mathrm{m}$. Thus, although nanobubbles were not explicitly considered by Og̃uz and Prosperetti, it can be assumed that viscous losses will also be a major dissipation mechanism in the case of the 100-nm-radius bubble trapped inside the hole. 


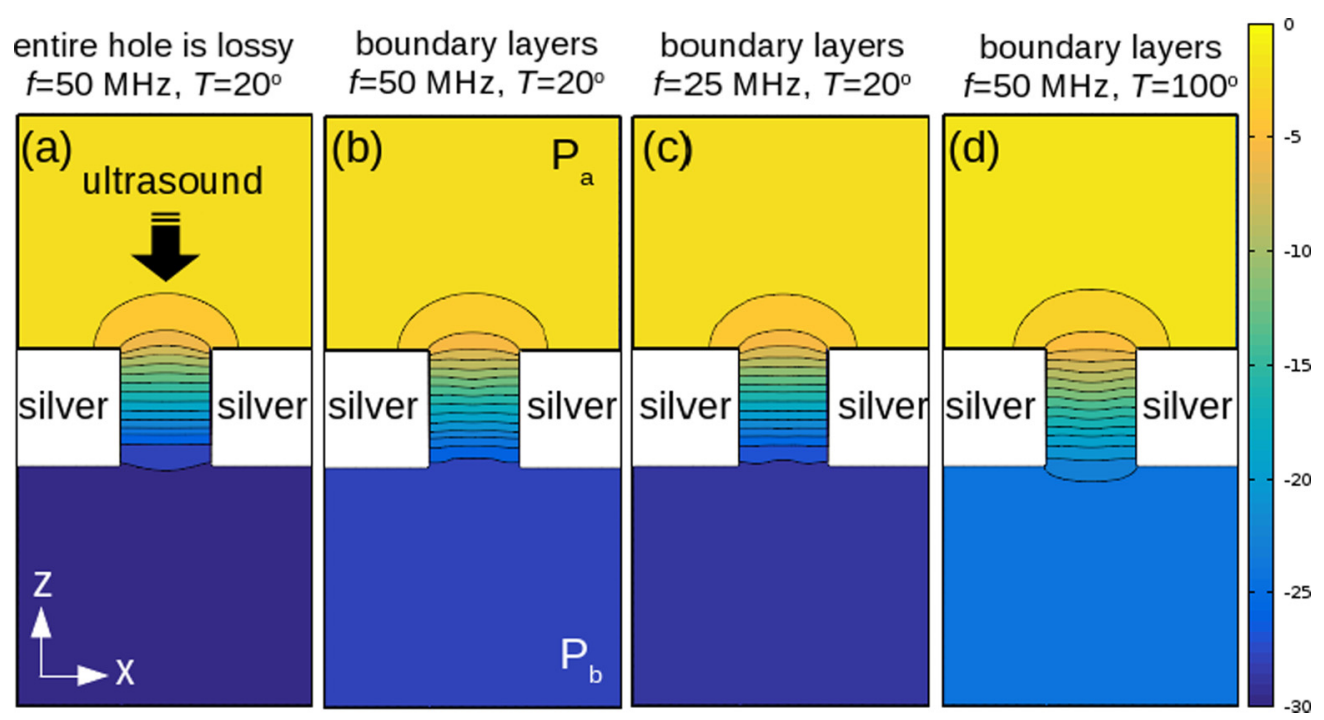

FIG. 7. Ultrasound pressure profile (dB-scale color bar) in the vertical cross section of the hole without the bubble. In contrast to the result in Fig. 4(a) obtained in the lossless approximation, here we take into account viscous and thermal losses. (a) Entire hole volume absorbs ultrasound through a combined viscous and thermal dissipation. (b) Absorption only happens near the walls of the hole in a layer with the thickness $d_{\mathrm{v}}=80 \mathrm{~nm}(f=50 \mathrm{MHz})$. (c) Absorption only happens near the walls of the hole in a layer with the thickness $d_{\mathrm{v}}=110 \mathrm{~nm}$ ( $f=25 \mathrm{MHz}$ ). (d) Absorption only happens near the walls of the hole in a layer with the thickness $d_{\mathrm{v}}=40 \mathrm{~nm}(f=50 \mathrm{MHz})$. Note that in (a)-(c) the temperature of water is $T=20^{\circ} \mathrm{C}$, but in (d) we model the scenario of $T=100^{\circ} \mathrm{C}$.

Og̃uz and Prosperetti also discuss the damping rate of the bubble oscillation [32]. In a fashion similar to the derivation of Eq. (2), they consider the bubble as a cylinder occupying a slice of the hole. But unlike in Eq. (2), the oscillation frequency is a complex number with the real part equal to the oscillation frequency inside the hole and the imaginary part equal to the viscous damping. Whereas the former value is already given by Eq. (2), the viscous damping is a function of the liquid viscosity. However, the resulting expression for the damping rate does not have a closed-form solution at arbitrary frequency. Consequently, Oguz and Prosperetti rely on approximations to estimate the relative input of viscous losses to the total damping of the oscillations. They conclude that the oscillations of a bubble inside a hole have the same damping rate as in the case of a bubble in an unbounded liquid.

Thus, the previous theory does not allow us to calculate a decrease in the bubble pulsation radius $R(t)$ due to viscous losses. However, it allows us to neglect for a moment the impact of the hole on the oscillation frequency of the bubble and discuss $R(t)$ of a bubble pulsating in an unbounded viscous liquid. It is known [34-36] that $R(t)$ of unbounded nanobubbles pulsating in a viscous liquid may adequately be described by a Rayleigh-Plesset equation with the viscous term. Consequently, we solve the following Rayleigh-Plesset equation $[21,33]$ :

$$
\rho\left(\ddot{R} R+1.5 \dot{R}^{2}\right)=p_{0}\left(R_{0} / R\right)^{3 \gamma}-p_{0}-P(t)-\frac{4 \eta \dot{R}}{R},
$$

where $\eta$ is the liquid viscosity. The solution of this equation will allow us to obtain $R(t)$ as a function of $\eta$. Returning to the scenario of the bubble inside the hole, according to the theory $R(t)$ should have the same dependence on $\eta$ but the oscillation frequency of the bubble will be $\sim 2$ times lower as predicted by Eq. (2).
We also note that in Eq. (3) we omitted the surface tension term $\sigma$, which allows us to demonstrate the effect of the liquid viscosity. The inclusion of $\sigma$ will lead to an additional decrease in the amplitude of the bubble pulsations.

Figure 8 compares the solutions of the lossless Eq. (1) (blue dashed curve) and Eq. (3) (red solid curve, $\eta=1.002 \times$ $10^{-3} \mathrm{~Pa}$ s at $20^{\circ} \mathrm{C}$ temperature). One can see that due to the viscous losses the bubble pulsation amplitude decreases by a factor of $\sim 4$. In addition, the oscillations present in the blue dashed curve at $t>0.1 \mu$ s disappear because the losses are

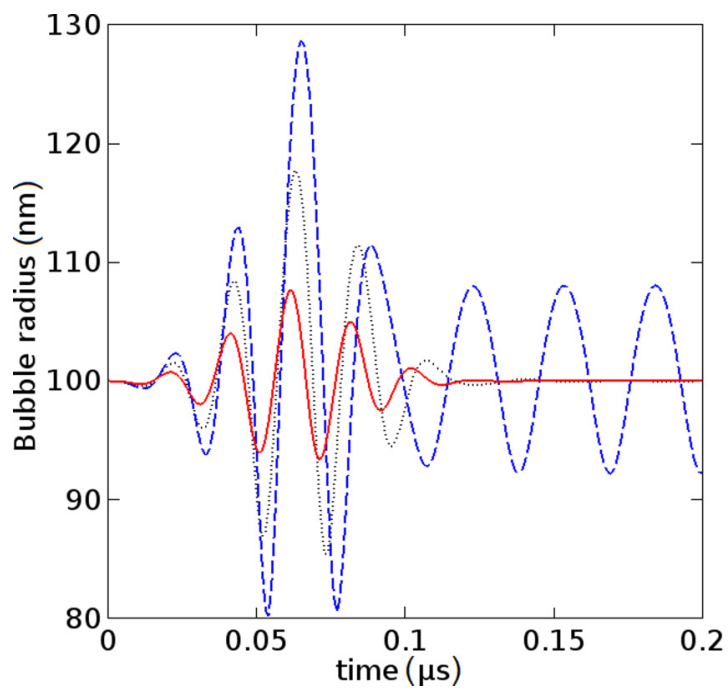

FIG. 8. Radial response $R(t)$ of a 100-nm-radius air bubble in water to the driving ultrasound pressure pulse with the center frequency $50 \mathrm{MHz}$. Red solid curve: $\eta=1.002 \times 10^{-3} \mathrm{P}$ a s at $20^{\circ} \mathrm{C}$. Black dotted curve: $\eta=0.282 \times 10^{-3} \mathrm{~Pa} \mathrm{~s}$ at $100^{\circ} \mathrm{C}$. Blue dashed curve: $\eta=0$ (lossless approximation). 
now taken into account. These changes will be translated into the corresponding decrease in the transmitted light intensity through the hole, in a fashion similar to the result in Fig. 6.

Finally we note that the propagation of light through the hole leads to optical absorption and Joule heating of the hole's walls [50], which eventually gives rise to an increase of the temperature of water inside the hole. It is known [51,52] that the viscosity of water gradually decreases as the temperature is increased to reach $\eta=0.282 \times 10^{-3} \mathrm{~Pa} \mathrm{~s}$ at $100^{\circ} \mathrm{C}$. Using this value of the viscosity as a limiting case, in Fig. 8 we show (black dotted line) that the amplitude of $R(t)$ becomes just $\sim 2$ times smaller than that in the lossless approximation.

Of course other factors will also influence the oscillations of the bubble when the temperature is changed. For example, an increase in the water temperature from 0 to $100{ }^{\circ} \mathrm{C}$ leads to a 1.3-fold decrease in the surface tension $\sigma$ and a concomitant increase in the amplitude of $R(t)$, which is a favorable effect. Moreover, a decrease in the viscosity of water may affect the stability of the bubble [52]. However, in our case this factor is of secondary importance, because a high bubble stability is mostly important in studies of single bubble sonoluminescence where the highest emitted light intensity corresponds to the largest achievable driving pressure [52].

We also note that the thermal conductivity $\kappa$ of water slightly increases as the temperature is increased, but at the same time the values of density $\rho$ and specific heat capacity at constant pressure $c_{\mathrm{p}}$ remain nearly unchanged [51]. Consequently, at $100^{\circ} \mathrm{C}$ the thickness of the viscous boundary layer decreases up to $d_{\mathrm{v}}=42 \mathrm{~nm}$ because the viscosity $\eta$ decreases, but the thickness of the thermal boundary layer remains nearly the same: $d_{\mathrm{h}}=32 \mathrm{~nm}$.

Furthermore, in our model the total viscosity of water is defined as $\eta_{\text {tot }}=\zeta+4 \eta / 3$, where $\zeta$ is the bulk viscosity. The value of $\zeta$ also quickly decreases as the temperature of water is increased [53]. This property may open up an additional degree of freedom in controlling the transmission of ultrasound through the hole by tuning the intensity of incident light. Our simulations confirm this possibility. Because data for $\zeta$ from [53] are not readily available for temperatures above $50{ }^{\circ} \mathrm{C}$, we extrapolate them to obtain $\zeta$ for water at $100^{\circ} \mathrm{C}$. Then we model the viscous layer thickness at $50 \mathrm{MHz}$ and $100{ }^{\circ} \mathrm{C}$ [Fig. 7(d)] and we show that the pressure inside the hole increases by $\sim 2.5$ times as compared with the case of water at $20^{\circ} \mathrm{C}$.

\section{CONCLUSIONS}

We have demonstrated a scheme for the control of light with ultrasound in a subwavelength hole loaded with a bubble. The scheme may find applications in novel optomechanic devices [18] and acousto-optical metamaterials such as phoxonic crystals [19], in which bubbles trapped inside the holes of the periodic photonic crystal pattern may be used to control the photonic band gap.

In a fashion similar to plasmonic subwavelength apertures [54], bubbles may also be employed to control light in a liquid-core optical fiber [42] or a liquid-filled dielectric slot waveguide [55]. Liquid-state devices such as optical fibers [42] and lenses [43] offer a number of potentially transformative advantages for microphotonic systems. Consequently, a large and growing body of research investigates novel liquid-based systems [56,57]. We believe that the integration of bubbles with subwavelength optical apertures may open up novel opportunities for the development of acousto-optical liquidstate photonic devices such as, e.g., optical hydrophones [41].

Our findings may also be useful in optofluidic laboratoryon-a-chip flow cytometry [58], sonoporation (ultrasoundmediated cell membrane disruption) [59], as well as in the development of new methods of biological cell manipulation [60]. For example, it has been proposed to both deform biological cells and monitor their deformations by the acoustic fields of an oscillating bubble, because the frequency of the natural oscillations of many cells is in the $\mathrm{MHz}$ frequency range and therefore the detection of such oscillations by Raman or Brillouin scattering is challenging [61]. However, for this promising technique to be implemented one has to control and quantify the oscillation of the bubble. Our results suggest that porous metal or metal-coated membranes may be used to all-optically sense the behavior of the bubble. A similar procedure may be useful in flow cytometry and sonoporation.

We expect that the presented scheme may also find applications in classical microwave waveguide theory and technique. This is possible because of a similarity between the plasmonic holes and circular microwave waveguides [4]. This similarity may be used to verify our theoretical prediction in the microwave frequency range. Structures tested at microwave frequencies are often easier to fabricate and experimentally investigate than nanostructures, and this approach was used in the past to verify theoretical predictions for dielectric nanoantennas [62]. A suitable candidate for the verification of our results may be a water-immersed brass plate with circular holes with millimeter-size diameter and depth [16]. From the point of view of electromagnetism, such holes may support guided modes and Fabry-Perot resonances in the microwave frequency range. On the other hand, such holes transmit acoustic waves and they may also be used to trap gas bubbles. Acoustically driven oscillations of the bubbles are expected to control microwave signals in a fashion similar to the control of light with bubbles.

The concept of acoustically controlled microwave transmission through a waveguide may also find practical applications in, for example, absorptive microwave switches [63]. Absorptive switches are required for high-power applications such as satellite communications, radars, and wide range Internet, because they absorb the incoming waves and do not generate potentially damaging reflections. Water-based waveguide absorptive switches [63] operate by appropriately placing water inside a waveguide to absorb the incident microwave signals. However, the volume of the water-air section of the waveguide has to be controlled manually, which allows us to achieve a static operation only. The use of an oscillating bubble may allow one to dynamically modify the air-water section of the waveguide, which is expected to result in a dynamic tuning of the absorption.

\section{ACKNOWLEDGMENTS}

This work was supported by Australian Research Council (ARC) through its Centre of Excellence for Nanoscale BioPhotonics (CE140100003) and LIEF program (LE160100051). 
This research was undertaken on the NCI National Facility in Canberra, Australia, which is supported by the Australian Commonwealth Government. The authors thank A. Llobera Adán, B. Gibson, and M. Hutchinson for useful discussions. The authors also thank an anonymous referee for bringing their attention to the boundary-layer effect on acoustic transmission through subwavelength holes.

\section{APPENDIX: OPTICAL AND ACOUSTIC FINITE-DIFFERENCE TIME-DOMAIN MODELS}

Simulations of the optical response of the bubble-hole structure require a numerical method capable of tackling geometries with interfaces between materials with disparate dielectric permittivities of silver, water, and air. The 3D optical finite-difference time-domain (FDTD) method is one of the numerical methods that offers this functionality [64]. The time-dependent Maxwell's equations (in partial differential form) are discretized using central-difference approximations to the space and time partial derivatives [64]. The resulting finite-difference equations are solved in a leapfrog manner. First, the electric field vector components in a volume of space are solved at a given instant in time. Then the magnetic field vector components in the same spatial volume are solved at the next instant in time. The process is repeated until the desired transient or steady-state electromagnetic field behavior is fully evolved.

In our customized optical FDTD software, the spatial resolution step equals $2 \mathrm{~nm}$ in all coordinate directions. The temporal resolution is chosen according to the Courant stability condition [64]. Optical perfectly matched layers (PMLs) are implemented to avoid unphysical reflections from the boundaries of the optical computational domain [64].

The Drude model $\epsilon_{\mathrm{Ag}}=\epsilon_{\infty}-\omega_{\mathrm{p}}^{2} /[\omega(\omega-i \gamma)]$ is required to simulate the frequency-dependent optical response of silver [64]. We implement the Drude model in the time domain by using the standard auxiliary differential equation method of treatment of the Drude dispersion [64]. The parameters of the Drude model for silver are: $\epsilon_{\infty}=4.96, \hbar \omega_{\mathrm{p}}=9.54 \mathrm{eV}$, and $\hbar \gamma=0.055 \mathrm{eV}$.

We also employ a 3D acoustic FDTD method [65] to model the transmission of ultrasound through the hole. The acoustic FDTD is conceptually similar to the optical FDTD, but the equations of linear acoustics are solved in a leapfrog fashion instead of Maxwell's equations [65]. The acoustic material parameters of water used in the FDTD model are [66]: density $\rho_{\text {water }}=1000 \mathrm{~kg} / \mathrm{m}^{3}$ and longitudinal speed of sound $v_{\text {water }}=1500 \mathrm{~m} / \mathrm{s}$. The density of air is $\rho_{\text {air }}=$ $1.2 \mathrm{~kg} / \mathrm{m}^{3}$ and the speed of sound in air is $v_{\text {air }}=343 \mathrm{~m} / \mathrm{s}$. When viscous and thermal losses are taken into account, the standard acoustic FDTD scheme is extended following the discussion in the Supplementary Information of [41] and we additionally use the following material parameters: the shear viscosity $\eta=1.002 \times 10^{-3} \mathrm{~Pa} \mathrm{~s}$, bulk viscosity $\zeta=3.09 \times 10^{-3}$ $\mathrm{Pa} \mathrm{s}$, specific heat capacity at constant pressure $c_{\mathrm{p}}=4.182$ $\times 10^{3} \mathrm{~J} /(\mathrm{kg} \mathrm{K})$ and constant volume $c_{\mathrm{v}}=c_{\mathrm{p}} / 1.33$, and thermal conductivity $\kappa=0.597 \mathrm{~W} /(\mathrm{m} \mathrm{K})$. Nonlinear phenomena are neglected in our acoustic FDTD model. Mur's absorbing boundary conditions are implemented to avoid unphysical reflections from the boundaries of the computational domain
$[64,65]$. The spatial resolution step used in the acoustic FDTD method equals $2 \mathrm{~nm}$.

The predictions of the acoustic 3D FDTD model were verified by using an elastodynamic 3D Virieux-FDTD method [67]. This software is capable of tackling geometries with interfaces between water, air, and silver. Conceptually, the Virieux-FDTD method is similar to the acoustic FDTD method, but it is based on the equations of elastodynamics that involve the components of the stress tensor $T$. We note that in our model in liquids, the normal stress components $T_{\mathrm{xx}}, T_{\mathrm{yy}}, T_{\mathrm{zz}}$ are the same values, equal to the opposite of the pressure of ultrasound, but the shear stress is zero. Thus, when only liquids are present in the elastodynamic model, this model effectively reduces to the acoustic FDTD model.

In addition to the material parameters of water and air described above, in our elastodynamic model we use the following material parameters of silver [66]: density $\rho_{\mathrm{Ag}}=$ $10490 \mathrm{~kg} / \mathrm{m}^{3}$, longitudinal speed of sound $v_{\mathrm{l}, \mathrm{Ag}}=3650 \mathrm{~m} / \mathrm{s}$, and transversal speed of sound $v_{\mathrm{t}, \mathrm{Ag}}=1610 \mathrm{~m} / \mathrm{s}$. The spatial
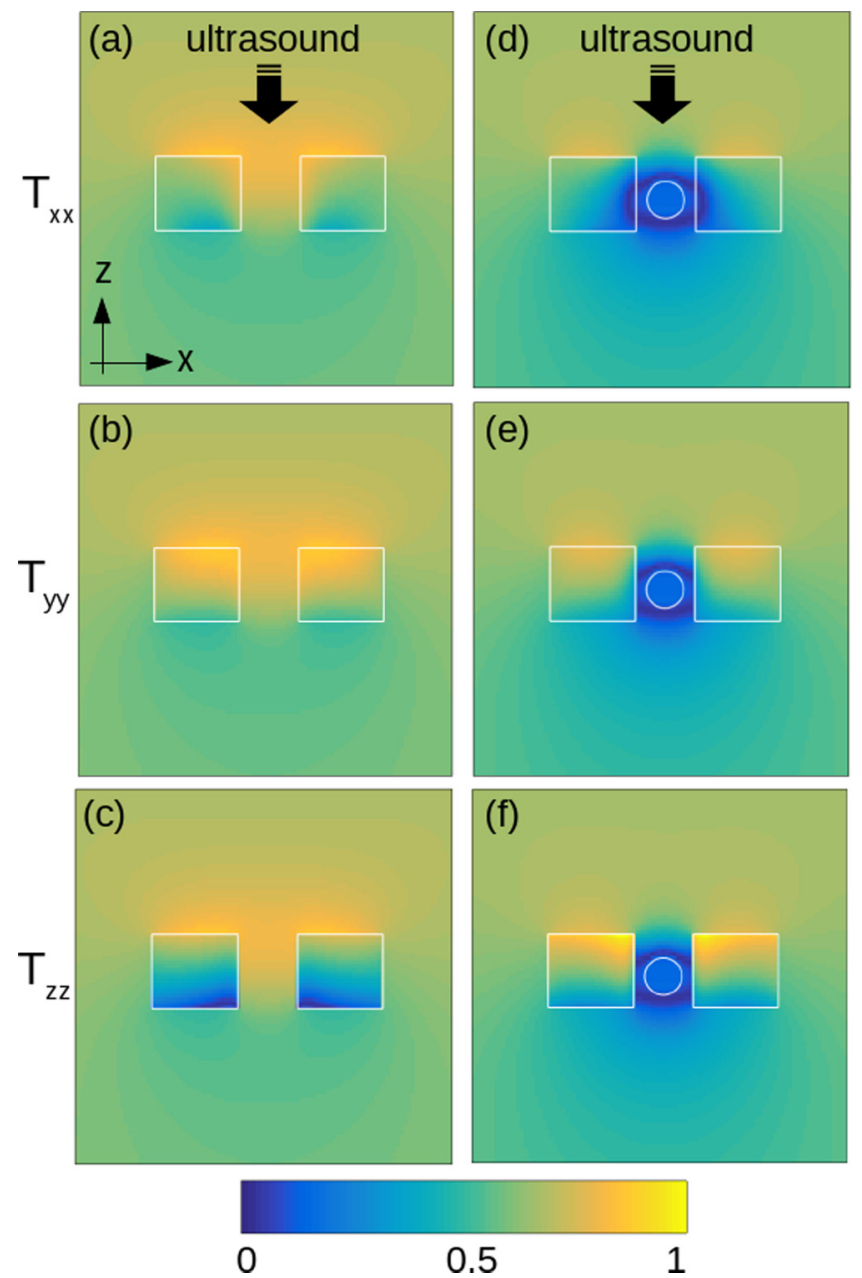

FIG. 9. (a)-(c) Amplitudes of the stress components $T_{\mathrm{xx}}, T_{\mathrm{yy}}$, and $T_{\mathrm{zz}}$ (linear color bar) in the vertical cross section of the hole without the bubble. The contours of the silver film are denoted by the white rectangles. Note that the simulated structure is slightly different from that in Fig. 4, as discussed in the text of the Appendix. (d)-(f) The same but in the presence of the bubble. 
resolution step used in the elastodynamic FDTD method equals $2 \mathrm{~nm}$. Absorption phenomena such as viscous and viscoelastic effects are neglected. Acoustic PMLs boundary conditions are used to truncate the computational domain [67].

Because our simulations numerically diverge when the silver film containing the hole contacts with the acoustic PMLs (which is probably due to a limitation of the software [67]), we have to reduce the area of the film such that it does not contact with the boundaries of the computational domain. As a result, the elastodynamic model differs from the acoustic model in Fig. 4 because the incident ultrasound can partially bend around the film. We cannot mitigate this artifact by significantly increasing the size of the computational domain because this manipulation leads to impractically large time and memory consuming simulations. However, in the following we demonstrate that this artifact does not interfere with our goal to confirm the validity of the acoustic model used in the main text.

In the left column of Fig. 9 we present the amplitudes of the $T_{\mathrm{xx}}, T_{\mathrm{yy}}$, and $T_{\mathrm{zz}}$ stress components plotted in the vertical cross section of the hole without the bubble. The contours of the silver film and hole are denoted by the white rectangles. For the reason mentioned above, we observe that ultrasound bends around the film and reaches the region below the film. Nevertheless, similar to the profile in Fig. 4(a), one can see that in the region above the film the pressure is higher than in the region below the film, because of the reflection of ultrasound from the film.

In the acoustic model used to produce Fig. 4(a), the incident ultrasound is reflected from the top surface of the silver film that is modeled as a perfectly rigid body. In contrast, in the elastodynamic model the ultrasound propagates inside the silver film. However, the stress amplitude inside the film quickly decreases because of the reflection from the bottom surface of the silver film. This can be seen by comparing the amplitudes inside the white rectangles in Fig. 9 (left column) with the amplitude in the region between the rectangles, which corresponds to the vertical cross section of the hole. Thus, we conclude that mostly ultrasound that leaks into the hole contributes to the transmission process through the hole. We notice that in the main text we arrived to the same conclusion by using the $3 \mathrm{D}$ acoustic model.

Finally, by using the elastodynamic FDTD model we simulate the scenario of the hole with an air bubble (right column of Fig. 9). As in the case of the hole without the bubble, we observe a good qualitative agreement between the results predicted with the acoustic [Fig. 4(b)] and elastodynamic models. For example, we observe that the pressure just above the hole decreases as compared with the case without the bubble, the pressure in front of the bubble decreases to zero, but overall the hole with the bubble remains partially transparent for ultrasound.
[1] C. Genet and T. W. Ebbesen, Nature (London) 445, 39 (2007).

[2] F. J. García de Abajo, Rev. Mod. Phys. 79, 1267 (2007).

[3] S. Carretero Palacios, Mechanism for Enhancing the Optical Transmission through a Single Subwavelength Hole (Prensas Universitarias de Zaragoza, Spain, 2011).

[4] I. S. Maksymov, M. Besbes, J.-P. Hugonin, J. Yang, A. Beveratos, I. Sagnes, I. Robert-Philip, and P. Lalanne, Phys. Rev. Lett. 105, 180502 (2010).

[5] I. Bulu, T. Babinec, B. Hausmann, J. T Choy, and M. Loncar, Opt. Express 19, 5268 (2011).

[6] R. Gordon, D. Sinton, K. L. Kavanagh, and A. G. Brolo, Acc. Chem. Res. 19, 1049 (2008).

[7] R. M. Cole, S. Mahajan, and J. J. Baumberg, Appl. Phys. Lett. 95, 154103 (2009).

[8] Y. J. Liu, E. S. P. Leong, B. Wang, and J. H. Teng, Plasmonics 6, 659 (2011).

[9] I. I. Smolyaninov, A. V. Zayats, A. Stanishevsky, and C. C. Davis, Phys. Rev. B 66, 205414 (2002).

[10] E. A. Shaner, J. G. Cederberg, and D. Wasserman, Appl. Phys. Lett. 91, 181110 (2007).

[11] I. S. Maksymov, Rev. Phys. 1, 36 (2016).

[12] D. Gérard, V. Laude, B. Sadani, A. Khelif, D. Van Labeke, and B. Guizal, Phys. Rev. B 76, 235427 (2007).

[13] I. S. Maksymov and M. Kostylev, J. Appl. Phys. 116, 173905 (2014).

[14] J. Christensen, A. I. Fernández-Domínguez, F. de León-Pérez, L. Martin-Moreno, and F. J. García-Vidal, Nat. Phys. 3, 851 (2007).

[15] H. Estrada, P. Candelas, A. Uris, F. Belmar, F. J. García de Abajo, and F. Meseguer, Phys. Rev. Lett. 101, 084302 (2008).
[16] V. Gómez-Lozano, C. Rubio, P. Candelas, A. Uris, and F. Belmar, Materials 9, 453 (2016).

[17] I. S. Maksymov, Nanomaterials 5, 577 (2015).

[18] M. Aspelmeyer, T. J. Kippenberg, and F. Marquardt, Rev. Mod. Phys. 86, 1391 (2014).

[19] A. Khelif and A. Adibi, Phononic Crystals: Fundamentals and Applications (Springer, New York, 2016).

[20] T. G. Leighton, The Acoustic Bubble (Academic, London, 1994).

[21] W. Lauterborn and T. Kurz, Rep. Prog. Phys. 73, 106501 (2010).

[22] H. Tsuge, Micro- and Nanobubbles: Fundamental and Applications (Pan Stanford, Singapore, 2014).

[23] D. Lapotko, Opt. Express 17, 2538 (2009).

[24] L. H. Thamdrup, F. Persson, H. Bruus, A. Kristensen, and H. Flyvbjerg, Appl. Phys. Lett. 91, 163505 (2007).

[25] K. Uehara and Y. Yano, IEEE Trans. Magn. 47, 2604 (2011).

[26] C. Zhao, Y. Liu, Y. Zhao, N. Fang, and T. J. Huang, Nat. Commun. 4, 2305 (2013).

[27] C. Duan, R. Karnik, M.-C. Lu, and A. Majumdar, PNAS 109, 3688 (2012).

[28] Y. Li, F. Nicoli, C. Chen, L. Lagae, G. Groeseneken, T. Stakenborg, H. W. Zandbergen, C. Dekker, P. Van Dorpe, and M. P. Jonsson, Nano Lett. 15, 776 (2015).

[29] T. Hofmann, D. Wallacher, J. Perlich, S. Koyiloth Vayalil, and P. Huber, Langmuir 32, 2928 (2016).

[30] H. S. White, B. Zhang, R. J. White, and E. N. Ervin, US Patent 2010/0025263 A1 (2010).

[31] S. R. German and T. L. Mega, US Patent 2016/0041122 A1 (2016).

[32] H. N. Og̃uz and A. Prosperetti, J. Acoust. Soc. Am. 103, 3301 (1998). 
[33] A. A. Doininkov, Phys. Fluids 14, 1420 (2002).

[34] R. Holyst, M. Litniewski, and P. Garstecki, Phys. Rev. E 82, 066309 (2010).

[35] M. G. González, X. Liu, R. Niessner, and C. Haisch, Appl. Phys. Lett. 96, 174104 (2010).

[36] Y. Mao and Y. Zhang, Nanosc. Microsc. Therm. 17, 79 (2013).

[37] N. W. Jang, S. M. Gracewski, B. Abrahamsen, T. Buttacio, R. Halm, and D. Dalecki, J. Acoust. Soc. Am. 126, EL34 (2009).

[38] D. T. Blackstock, Fundamentals of Physical Acoustics (Wiley, New York, 2000).

[39] F. J. García de Abajo, H. Estrada, and F. Meseguer, New J. Phys. 11, 093013 (2009).

[40] A. E. Miroshnichenko, S. Flach, and Yu. S. Kivshar, Rev. Mod. Phys. 82, 2257 (2010).

[41] I. S. Maksymov and A. D. Greentree, Sci. Rep. 6, 32892 (2016).

[42] R. Altkorn, I. Koev, R. P. Van Duyne, and M. Litorja, Appl. Opt. 36, 8992 (1997).

[43] T. Krupenkin, S. Yang, and P. Mach, Appl. Phys. Lett. 82, 316 (2003).

[44] Y. A. Ilinskii, E. A. Zabolotskaya, T. A. Hay, and M. F. Hamilton, J. Acoust. Soc. Am. 132, 1346 (2012).

[45] D. Lohse and X. Zhang, Rev. Mod. Phys. 87, 981 (2015).

[46] P. M. Morse and K. Uno Ingard, Theoretical Acoustics (McGraw-Hill, New York, 1968)

[47] G. P. Ward, R. K. Lovelock, A. R. J. Murray, A. P. Hibbins, J. R. Sambles, and J. D. Smith, Phys. Rev. Lett. 115, 044302 (2015).

[48] D. Homentcovschi, R. N. Miles, and L. Tan, J. Acoust. Soc. Am. 117, 2761 (2005).

[49] D. Homentcovschi and R. N. Miles, Wave Motion 45, 191 (2008).

[50] G. Baffou, R. Quidant, and F. J. García de Abajo, ACS Nano 4, 709 (2010).

[51] J. V. Sengers and J. T. R. Watson, J. Phys. Chem. Ref. Data 15, 1291 (1986).
[52] S. Hilgenfeldt, D. Lohse, and W. C. Moss, Phys. Rev. Lett. 80, 1332 (1998).

[53] M. J. Holmes, N. G. Parker, and M. J. W. Povey, J. Phys.: Conf. Series 269, 012011 (2011).

[54] I. S. Maksymov and A. D. Greentree, arXiv:1610.04721 [Opt. Exp. (to be published)].

[55] C. A. Barrios, Sensors 9, 4751 (2009).

[56] E. Eunjung Jung and D. Erickson, Lab Chip 12, 2575 (2012).

[57] C. L. Phillips, E. Jankowski, B. Jyoti Krishnatreya, K. V. Edmond, S. Sacanna, D. G. Grier, D. J. Pine, and S. C. Glotzer, Soft Matter 10, 7468 (2014).

[58] S. H. Cho, J. M. Godin, C.-H. Chen, W. Qiao, H. Lee, and Y.-H. Lo, Biomicrofluidics 4, 043001 (2010).

[59] Z. Fan, H. Liu, M. Mayer, and C. X. Deng, PNAS 109, 16486 (2012).

[60] P. V. Zinin and J. S. Allen III, Phys. Rev. E 79, 021910 (2009).

[61] M. G. Beghi, A. G. Every, and P. V. Zinin, in Ultrasonic Nondestructive Evaluation: Engineering and Biological Material Characterization, edited by T. Kundu (CRC, Boca Raton, 2004), p. 581.

[62] A. E. Krasnok, I. S. Maksymov, A. I. Denisyuk, P. A. Belov, A. E. Miroshnichenko, C. R. Simovski, and Yu. S. Kivshar, Phys. Usp. 56, 539 (2013).

[63] C.-H. Chen and D. Peroulis, IEEE Trans. Microwave Theory Tech. 57, 2038 (2009).

[64] A. Taflove and S. C. Hagness, Computational Electrodynamics: The Finite-Difference Time-Domain Method, 3rd ed. (Artech House, Norwood, MA, 2005).

[65] D. M. Sullivan, Electromagnetic Simulation Using the FDTD Method (IEEE, NJ, 2000).

[66] D. E. Gray, American Institute of Physics Handbook (McGrawHill, New York, 1957).

[67] E. Bossy, User's Guide for SimSonic3D (2012), available at: http://www.simsonic.fr. (Accessed June 4, 2016.) 